\title{
Synchronization in disordered oscillator lattices: Nonequilibrium phase transition for driven-dissipative bosons
}

\author{
John P. Moroney (10) and Paul R. Eastham (1)* \\ School of Physics, Trinity College Dublin, Dublin 2, Ireland
}

(Received 15 January 2021; accepted 22 October 2021; published 1 November 2021)

\begin{abstract}
We show that lattices of phase oscillators with random natural frequencies undergo a transition from a desynchronized to a synchronized state for dimensions $d<4$. The oscillators are described by a generalization of the nearest-neighbor Kuramoto model with an additional cosine coupling term. This model may be derived from the complex Ginzburg-Landau equations for a lattice of driven-dissipative Bose-Einstein condensates of exciton polaritons. We derive phase diagrams that classify the desynchronized and synchronized states, focusing on the behavior in one and two dimensions. This is achieved by outlining the connection of the oscillator model to the quantum description of localization of a particle in a random potential through a mapping to a modified Kardar-Parisi-Zhang equation. Our results indicate that synchronization in coupled polariton condensates and other examples of low-dimensional lattices of coupled oscillators is not destroyed by randomness in their natural frequencies.
\end{abstract}

DOI: 10.1103/PhysRevResearch.3.043092

\section{INTRODUCTION}

Synchronization of coupled oscillators is a phenomenon that appears regularly throughout nature. Many seemingly unrelated systems which exhibit repetitive behaviors, such as clocks, pacemaker cells in the heart, or a swarm of pulsing fireflies, are seen to undergo transitions from initial randomness to an ordered state [1,2]. A celebrated model of such synchronization phenomena was introduced by Kuramoto $[3,4]$. With all-to-all couplings, the Kuramoto model undergoes a phase transition, from a desynchronized state at weak coupling to a synchronized state, with a single frequency, at strong coupling. However, this global frequency ordering does not occur for oscillators coupled only to their neighbors on a finite-dimensional lattice [5-7].

Synchronization plays an important role in the physics of Bose-Einstein condensation in driven-dissipative Bose gases. Such condensation has been realized for exciton polaritons [8], which are bosonic quasiparticles formed from the strong coupling of excitons and photons in semiconductor microcavities. The condensates are described by a single macroscopic wave function [9], giving rise to phenomena such as superfluidity, Josephson oscillations [10], and quantized vortices and enabling applications such as analog simulation $[11,12]$. In these systems, the decay of the polaritons is offset by gain from the pump, and condensation occurs in a nonequilibrium steady state. Above threshold, the non-

\footnotetext{
*easthamp@tcd.ie

Published by the American Physical Society under the terms of the Creative Commons Attribution 4.0 International license. Further distribution of this work must maintain attribution to the author(s) and the published article's title, journal citation, and DOI.
}

linear gain fixes the density of the condensate, forming an autonomous phase oscillator whose frequency corresponds to the energy of the condensate. Spatially separated condensates can form in the random potentials arising from disorder in the samples [13] and in engineered potentials such as lattices $[11,14]$. If the condensates are well separated, they oscillate independently, but their mutual coupling can lead them to synchronize to a common frequency and phase [15]. Such effects have been observed for polaritons trapped in double-well potentials $[10,15]$, in the intrinsic random potential of the samples [13,16], and in the wells of a weakly disordered two-dimensional (2D) lattice [14]. We note that localized condensates are not necessarily trapped in potential wells: nonuniform pumping can also produce condensates at the peaks of the potential, which can synchronize with one another $[11,17]$. Such condensates are propagating states and so can have a significant dissipative part to their coupling $[18,19]$.

In this paper, we study the ordered states of drivendissipative bosons that are trapped in the wells of a disordered lattice potential. We focus on the $2 \mathrm{D}$ case, as realized in a recent experiment [14]. We seek to understand the phase diagram and phase transitions beyond the equilibrium case in which there is a superfluid-insulator transition [20]. The nonequilibrium problem is described by a lattice of coupled oscillators, with disordered frequencies, suggesting that there may be a frequency-ordering transition or a frequency- and phase-ordering one. Ordered states are, however, not expected for Kuramoto oscillators with random frequencies in 2D [5-7]. This is consistent with previous predictions [21] that there is no superfluid-insulator transition in driven-dissipative condensates.

We investigate synchronization in a lattice of drivendissipative condensates with random natural frequencies. Such a lattice is described by a generalization of the 
nearest-neighbor Kuramoto model that includes an additional coupling term that is even in the relative phases. Such models have previously been considered [22,23] in the case where the natural frequencies are identical and frequency synchronization is expected, but the phases may become disordered due to the space- and time-dependent noise associated with gain and loss [24-27]. We consider the opposite limit of random natural frequencies but negligible time-dependent noise. We find that the non-Kuramoto coupling has a significant effect: it allows synchronization to occur in large lattices, formed from macroscopic numbers of oscillators, for dimensions $d<4$. The resulting state has a single frequency and phase correlations which extend over the entire lattice.

Our argument involves relating the oscillator model to a Kardar-Parisi-Zhang (KPZ) equation [28] with timeindependent noise [29-33] and then to an imaginary-time Schrödinger equation with a random potential. This allows us to derive the phase boundary for synchronization and characterize the frequency and phase profiles. These analytical predictions agree with numerical simulations in one (1D) and 2D. Our results show that, contrary to the case of Kuramoto oscillators, there is global frequency synchronization in large lattices of driven-dissipative condensates and that there is a region of parameters where this long-range coherence is robust against (static) disorder. Our conclusions apply more generally to coupled oscillator systems, implying there are other settings [1] in which this synchronization could occur.

\section{DYNAMICS OF NONEQUILIBRIUM CONDENSATES}

Under the requisite conditions [34], a polariton condensate may be described by a driven-dissipative Gross-Pitaevskii equation [35]:

$$
\begin{aligned}
i \frac{\partial \Psi}{\partial t}= & {\left[-\frac{1}{2 m} \nabla^{2}+V_{0}(\mathbf{r})\right] \Psi+U_{0}|\Psi|^{2} \Psi } \\
& +i\left[g_{0}(\mathbf{r})-\Gamma_{0}|\Psi|^{2}\right] \Psi,
\end{aligned}
$$

where $U_{0}$ is the polariton-polariton interaction strength, while $g_{0}(\mathbf{r})$ and $\Gamma_{0}$ account for the linear gain-resulting from the difference of pumping and decay-and the gain saturation. Here, $V_{0}(\mathbf{r})$ is a confining potential, which can arise from the repulsive interaction with the exciton reservoir, etching, deposition, and the intrinsic disorder in the sample [14,36]. Here and in the remainder of this paper, we set $\hbar=1$. Equation (1) is also known as the complex Ginzburg-Landau equation and is ubiquitous in descriptions of oscillatory media and nonlinear dynamical systems [37].

We consider a lattice of $N=L^{d}$ condensates, each of which forms in the ground state of one well of a lattice potential. We consider, for definiteness, a simple $1 \mathrm{D}$ chain and a 2D square lattice, as realized in a recent experiment [14]. In that experiment, a spatially patterned pump beam populates the exciton reservoir, forming the confining lattice potential. This potential is superimposed on the random potential due to disorder in the sample, leading to small variations in the energy from site to site.

We model this by expanding the macroscopic wave function over the basis set of wave functions localized in individual wells [38]. Assuming that the overlap between neighboring wave functions is small, so that direct overlap terms can be neglected, results in $N$ coupled equations for the amplitudes of each site $\psi_{k}$ :

$$
i \frac{\partial \psi_{k}}{\partial t}=\left[\epsilon_{k}+U\left|\psi_{k}\right|^{2}+i\left(g-\Gamma\left|\psi_{k}\right|^{2}\right)\right] \psi_{k}-\sum_{<l>} J_{k l} \psi_{l} .
$$

Here, $\epsilon_{k}$ is the energy of the condensate on a given lattice site, $U, g$, and $\Gamma$ are the interaction strength and gain coefficients, and the sum is over nearest neighbors. Also, $J_{k l}$ is the matrix element describing tunneling between neighboring sites. It can, in general, have imaginary parts, describing dissipative couplings between condensates [18]. However, this is not the case here, as we are considering condensation in bound states, whose real-valued wave functions are assumed to have negligible direct overlap. Further details of the derivation of Eq. (2) can be found in Appendix A.

Since the pumping, which produces the largest contribution to the potential and controls the gain on each site, has the periodicity of the lattice, the quantities $J_{k l} \approx J, U, g$, and $\Gamma$ vary little from site to site, and we treat them as constant. The site energies $\epsilon_{k}$, however, vary randomly from site to site due to the random contribution to the potential from the sample. We use the standard deviation $\sigma$ of the energies $\epsilon_{k}$ to characterize the strength of the disorder.

\section{COUPLED OSCILLATOR MODEL}

To examine synchronization in this system, we reparameterize our equations in terms of density and phase with the substitution $\psi_{k}=\sqrt{n_{k}} \exp \left(-i \theta_{k}\right)$. Well above threshold, the density variation between condensates is small, $\delta n_{k l}=$ $n_{k}-n_{l} \ll n_{k}$. Furthermore, the fast relaxation of the densities allows for their adiabatic elimination. This gives

$$
\frac{\partial \theta_{k}}{\partial t}=\epsilon_{k}+\frac{g}{\alpha}+J \sum_{<l>}\left[\frac{1}{\alpha} \sin \left(\theta_{l}-\theta_{k}\right)-\cos \left(\theta_{l}-\theta_{k}\right)\right] \text {. }
$$

Further details of this derivation can be found in Appendix B. We have introduced the dimensionless parameter $\alpha \equiv \Gamma / U$. The overall blueshift $g / \alpha$ can be removed by a redefinition of the zero of frequency. We can choose $1 / \sigma$ as our unit of time, so that the solutions to Eq. (3) are controlled by two dimensionless parameters $\alpha$ and $J / \sigma$.

Equation (3) is an equation for the phase dynamics in a system of coupled self-sustained oscillators. If we neglect the cosine term in the sum, it is the nearest-neighbor Kuramoto model [5]. In general, we expect that the existence of a globally synchronized solution-whereby the oscillators rotate at a common frequency, $\dot{\theta}_{1}=\dot{\theta}_{2}=\cdots=\dot{\theta}_{N}=\Omega$-will be dependent on the magnitudes of the tunneling and the spread of onsite energies. If there is no tunneling $(J=0)$, each oscillator will rotate at its blueshifted natural frequency. When $J$ is large relative to the spread of the natural frequencies, however, the frequency of each oscillator is strongly affected by the phase difference between it and its neighbors, and this mechanism can bring about synchronization. Despite this, it has been shown that extended lattices of Kuramoto oscillators, with random onsite energies and nearest-neighbor couplings, 




FIG. 1. Probability of synchronization of chains of condensates of varying lengths, determined by solving Eq. (3) with $\alpha=1$. Each probability is estimated from 100 disorder realizations, each simulated up to a time $t \sigma=1.8 \times 10^{4}$. The curves are fits to the Gumbel distribution.

do not exhibit synchronization [5-7]. In 1D, the probability of synchronization can be calculated as a function of $N$, and a coupling strength of order $\sqrt{N}$ is required for a synchronized solution [6].

The presence of the cosine term in the coupling function of our model has a significant effect, however. Unlike the Kuramoto model, our coupling is nonodd in its arguments, and it has been suggested that this may bring about synchronization more readily $[5,39,40]$. Numerical simulations of Eq. (3) illustrate that this is indeed the case. Figure 1 shows the probability of synchronization for chains of condensates of various lengths with normally distributed onsite energies. This probability is determined by calculating the average frequency of each oscillator from its phases at two times, one a short time after the initial transient behavior has decayed and the other a long time later. If each bond between neighboring sites has a frequency difference less than the smallest numerically resolvable frequency, the configuration is considered synchronized. Here, $P_{\text {sync }}(J)$ is then estimated from the fraction of synchronized results arising over many disorder realizations. We see that the probability of synchronization varies from almost zero to almost one over a range of $J$. The width of this range is nonzero because different realizations synchronize at slightly different tunneling strengths. More importantly, the position of the center of this range, which we use to define a typical critical tunneling strength $J_{c}$ such that $P_{\text {sync }}\left(J_{c}\right)=0.5$, is almost independent of the system size. This strongly suggests that there is a synchronization transition in the limit of thermodynamically large systems. This would be a nonequilibrium phase transition, at which the steady state of a system in the thermodynamic limit changes character, giving rise to singularities in its properties. Such behavior is markedly different from that of Kuramoto oscillators, where $J_{c}$ scales with $\sqrt{N}$, so that synchronization occurs only in small systems.

\section{CONTINUUM THEORY}

To understand the synchronization transition and the nature of the synchronized states, we consider the continuum form of Eq. (3) in the limit where the phase differences between neighboring sites are small. This will be the case in a synchronized state for weak disorder. Expanding the trigonometric functions to second order and taking the continuum limit, we have

$$
\frac{\partial \theta(\mathbf{x}, t)}{\partial t}=\epsilon(\mathbf{x})+\frac{J a^{2}}{\alpha} \nabla^{2} \theta+J a^{2}(\nabla \theta)^{2},
$$

where $a$ is the lattice constant, which will be set to one in the following. A uniform energy shift $g / \alpha-2 J d$ has been absorbed in the definition of $\theta(\mathbf{x}, t), d$ being the dimensionality of the system. Equation (4) is like the KPZ equation but has a time-independent noise term [28,32]. The connection between the complex Ginzburg-Landau equation and the conventional KPZ equation, with spatiotemporal noise, has been made in previous studies of polariton condensation [24-27], and indeed lattice models similar to Eq. (3) have also been studied $[22,23,41]$. Those works consider noise associated with gain and loss rather than that due to a random potential. The form of the noise in Eq. (4) results from our consideration of purely spatial disorder and leads to different universal behavior. The phase $\theta(x, t)$ behaves like the height of an interface, with a growth rate $\epsilon(\mathbf{x})$ which is random in space but not in time.

A Cole-Hopf transformation:

$$
Z(\mathbf{x}, t)=\exp (\alpha \theta),
$$

enables us to write the KPZ equation as

$$
\frac{\partial Z(\mathbf{x}, t)}{\partial t}=\frac{J}{\alpha} \nabla^{2} Z+\alpha \epsilon(\mathbf{x}) Z=-\hat{H} Z .
$$

This is the imaginary-time Schrödinger equation for a particle of mass $\alpha / 2 J$ in a random potential $V(\mathbf{x})=-\alpha \epsilon(\mathbf{x})$. In this form, it also describes the evolution of a population with diffusion and random autocatalytic amplification [29,30] and the partition function for a directed polymer in a random potential $[31,33]$. The general solution of Eq. (6) can be expressed in terms of the eigenstates of $\hat{H}, \varphi_{n}(\mathbf{x})$, with energies $E_{n}$ :

$$
Z(\mathbf{x}, t)=\sum_{n} c_{n} \varphi_{n}(\mathbf{x}) e^{-E_{n} t},
$$

which approaches the ground state wave function of the potential $V(\mathbf{x})$ for $t \rightarrow \infty$. For long but finite times, $Z(\mathbf{x}, t)$ will have contributions from a small number of low-energy states. In dimensions $d<4$, these low-lying states will be localized $\varphi_{n} \sim \exp \left(-\left|\mathbf{x}-\mathbf{x}_{n}\right| / \zeta\right)$ at some dilute positions $\mathbf{x}_{n}$, with the localization length $\zeta$ given by balancing the kinetic and potential terms in Eq. (6): $D / \zeta^{2} \sim u_{L} / \zeta^{d / 2}$ [30]. Here, $D=J / \alpha$, and $u_{L}=\alpha \sigma$, so we have

$$
\zeta \sim\left(\frac{D}{u_{L}}\right)^{2 /(4-d)}=\left(\frac{J}{\alpha^{2} \sigma}\right)^{2 /(4-d)} .
$$

In Figs. 2 and 3, we show some phase and frequency profiles obtained by solving the coupled oscillator model, Eq. (3). These solutions agree qualitatively with those of the continuum model, given by Eqs. (5) and (7). At short times, multiple peaks are evident in the phase profile, each of which corresponds to a localized, low-energy state of the Hamiltonian $\hat{H}$. The corresponding energies appear as plateaus in the frequency profile. The low-lying states have 


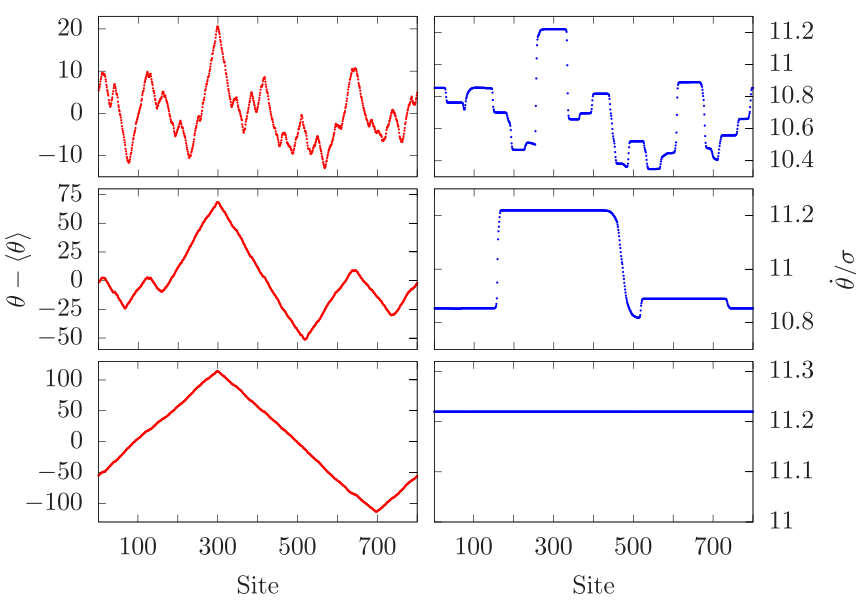

FIG. 2. Phase (left column) and frequency (right column) profiles in a chain of 800 coupled oscillators, with $J / \sigma=3.33$ and $\alpha=1$. The oscillators initially have a uniform phase. The profiles are shown after times $t \sigma=100$ (top row), 600 (middle row), and 24000 (bottom row).

negative energies, and so they grow in magnitude, corresponding to a steadily increasing phase in the region controlled by each localization center. The ground state, with the lowest energy, grows fastest, and eventually dominates the solution, giving a solution with a single peak in the phase profile and a single frequency (corresponding to the ground state energy of $\hat{H})$.

\section{PHASE DIAGRAM}

The solutions to the continuum model always synchronize, given sufficient time, but this is not the case for the original model. To investigate the conditions for synchronization, we
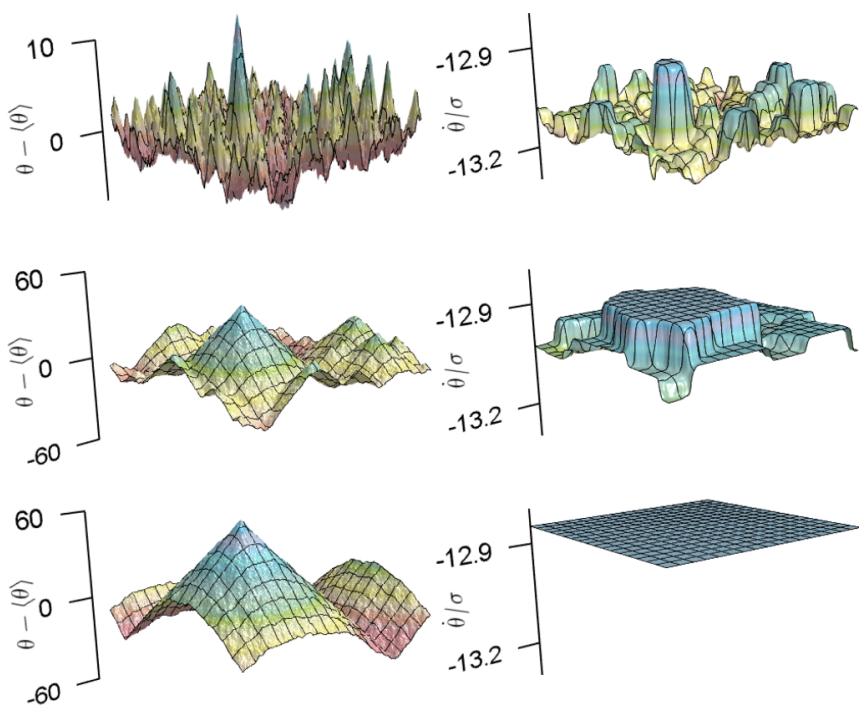

FIG. 3. Phase (left column) and frequency (right column) profiles in a two-dimensional lattice of $512 \times 512$ coupled oscillators, with $J / \sigma=3.33$ and $\alpha=1$. The oscillators initially have a uniform phase. The profiles are shown after times $t \sigma=50$ (top row), 300 (middle row), and 1700 (bottom row).
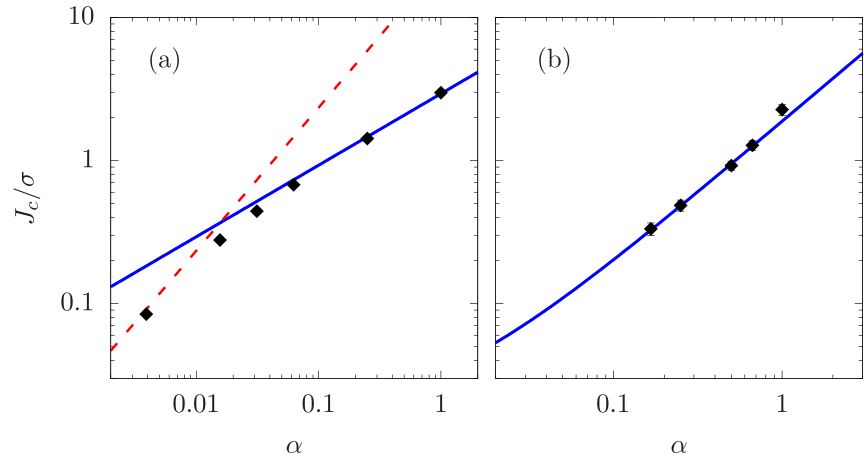

FIG. 4. Phase boundary for synchronization for (a) a onedimensional (1D) chain of 800 oscillators, and (b) a two-dimensional (2D) square lattice of $128 \times 128$ oscillators. $J_{c}$ is defined as the value of $J$ corresponding to a probability of synchronization of 0.5 . The points are numerical values, computed as discussed in the text. The solid lines show fits to the predicted slopes of (a) 0.5 or (b) 1 . The dashed line in (a) is the phase boundary for synchronization for a chain of 800 Kuramoto oscillators.

consider the phase gradients. Since the solutions to Eq. (6) are formed from exponentially localized states with localization length $\zeta$, we have, from Eqs. (5) and (8),

$$
|\nabla \theta| \sim \frac{1}{\alpha \zeta} \sim\left[\alpha^{d}\left(\frac{\sigma}{J}\right)^{2}\right]^{1 /(4-d)} .
$$

The continuum model does not impose a limit on these gradients. However, it only captures the behavior of the original model when $|\nabla \theta| \lesssim 1$. Otherwise, the periodicity and limited range of the trigonometric functions are important, and we cannot expand them in a power series. This argument gives

$$
J_{c} \sim \sigma \alpha^{d / 2}
$$

as the phase boundary for synchronization. This condition is relevant for $d<4$, as localized solutions to Eq. (6) are not guaranteed otherwise.

The dependence of $J_{c}$ on $\alpha$ given by Eq. (10) is confirmed by simulations for lattices in 1D and 2D, as shown in Fig. 4. We simulate Eq. (3) to obtain $P_{\text {sync }}(J)$, fit the resulting functions to a Gumbel extreme-value distribution, and use the fitted parameters to find $J_{c}$. We note that this distribution is expected from the analysis above because the probability that a given sample is synchronized is the probability that $\hat{H}$ has a ground-state localization length greater than a certain value or, equivalently, that the magnitude of its ground-state energy is less than a certain value. Since the ground-state energy is an extreme value - the lowest energy among the states - this quantity is the cumulative distribution function for an extreme-value distribution, and since the density of states in a band tail vanishes exponentially, it should be the Gumbel one. As can be seen in Fig. 1, this does indeed give a good description of our results.

The points in Fig. 4 show the values of $J_{c}$ determined from simulations. The solid curves show the predicted squareroot (1D) and linear (2D) dependencies, which are in good agreement with the data. On the 1D phase diagram, we also show the prediction of the conventional Kuramoto model for a chain of $N=800$ sites, for which $J_{c} \sim \sigma \alpha \sqrt{N}$ [6]. While the 
critical coupling in that case, without the cosine term, diverges for large $N$, for a small system, it can nonetheless lie below Eq. (10) at small $\alpha$. This explains the crossover seen at small $\alpha$ in Fig. 4(a). This is the standard finite-sized behavior if the cosine term is relevant in the renormalization group sense: such terms, even if they are small at the lattice scale, grow with distance and control the physics in sufficiently large systems. In small systems, however, the growth can be cut off by the system size.

Although our numerical results agree with Eq. (10) for $\alpha \lesssim 1$, they disagree in the opposite regime, where we find large sample-to-sample fluctuations and many states which are desynchronized even at large $J$. This may be related to dynamical instabilities in that regime [22,23]. In any case, Eq. (8) holds only in the weak disorder regime where the localization length $\zeta$ is larger than the lattice spacing $a=1$, and the localization length at the transition is, from Eqs. (8) and (10), $\zeta_{c} \sim 1 / \alpha$. More generally, $\zeta_{c}>1$ is needed so that space can be treated as continuous, as in Eq. (4), at the phase boundary. Importantly, the existence of a synchronization transition in the continuum regime implies the phenomenon is universal and independent of the details of the lattice or disorder. We note that a synchronization transition for 1D chains of oscillators with nonodd nearest-neighbor coupling was previously identified by Östborn [40]. That analysis, however, predicts a critical $J_{c}$ which differs from Eq. (10) and does not agree well with our numerical results.

\section{DISCUSSION}

Our expression for the ground-state localization length, Eq. (8), comes from a standard argument [30] equating the kinetic energy and the depth of a typical potential well $u_{L} / \zeta^{d / 2}$. In principle, one should use not the depth of a typical well but that of the deepest of all the $\sim(L / \zeta)^{d}$ wells of size $\sim \zeta$. For a normal distribution of well depths, these differ by a factor $\sqrt{d \ln L / \zeta}$, which seems to lead to the surprising conclusion that the localization length vanishes in the thermodynamic limit. This would mean, in our analysis, that $J_{c}$ diverges in this limit, and there is ultimately no synchronization. However, one should bear in mind that physical systems are never infinite, although they may be very large. As was pointed out in the careful analysis of Ref. [30], this factor grows extremely slowly with $N=L^{d}$, and it is not large, even in a macroscopic system. For example, for $(L / \zeta)^{d}=$ $N_{A} \sim 10^{23}$, we have $\sqrt{d \ln L / \zeta} \sim 7$. Thus, it does not prevent synchronization in the limit of a large physical system. One should bear in mind also that this formal divergence arises from the assumption of an unbounded distribution of well depths, which does not occur in practice.

It is interesting to compare our analysis and results with those for the nearest-neighbor Kuramoto model, for which complete synchronization can be ruled out on the grounds of self-consistency when $d \leqslant 2$ [5]. The argument involves expanding the trigonometric functions in an assumed synchronized solution, as we did to obtain Eq. (4). However, as Kuramoto oscillators have an odd (sine) coupling function, the nonlinear term in Eq. (4) would be absent. This means that the continuum theory for Kuramoto oscillators is a linear interface-growth model related to the Edwards-Wilkinson equation [7] rather than a nonlinear one related to the KPZ equation. The phase-phase correlation functions in the linear case diverge with the lattice size, for any fixed separation, when $d \leqslant 2$. This divergence is not consistent with the expansion of the trigonometric functions, so the assumed synchronized state does not occur.

Physical insight into the difference between the models can be obtained by considering the behavior of the phase gradients. For Kuramoto oscillators, the phase gradients in a synchronized solution can be obtained using an Imry-Ma [42] argument, like that previously outlined for the disordered polariton condensate in Ref. [21]. We consider Eq. (4), without the final term, and integrate over a region of space of linear dimension $\mathcal{L}$. The first term on the right-hand side is a random walk, which scales with the size of the region as $\mathcal{L}^{d / 2}$. It is related, in the polariton case, to the net random current generated in the region. This must balance the second term, which is related by the divergence theorem to the current flowing through the boundary. We see this second term is, at most, of order $|\nabla \theta| \mathcal{L}^{d-1}$. Comparing the two terms, we have that the phase gradients at the boundary of the region scale at least as fast as $|\nabla \theta| \sim \mathcal{L}^{(2-d) / 2}$. This implies they diverge for $d=1$ and is consistent with a logarithmic divergence in $d=2$ [5]. It contrasts with the result for the phase gradients in the model with the cosine coupling, Eq. (9), which are independent of the size of the region considered. Thus, in that case, there can be solutions with $|\nabla \theta| \lesssim 1$ everywhere, and the synchronized state is self-consistent.

We note that these self-consistency arguments do not rule out complete synchronization for lattices of Kuramoto oscillators in three or more dimensions. However, Strogatz and Mirollo have shown that this does not occur in any finite dimension [6]. They also do not rule out the possibility of partially synchronized states, where a macroscopic number of oscillators entrain to a single frequency. Numerical studies suggest that this does occur for Kuramoto oscillators but only in three or more dimensions [7].

\section{CONCLUSIONS}

We have shown that a model for coupled phase oscillators, which describes a disordered lattice of polariton condensates, has a synchronized state that survives in the limit of a macroscopic number of oscillators. At the critical coupling strength, given by Eq. (10), tunneling between condensates overcomes the localizing effects of the random potential, leading to a state with a single frequency and a stable phase profile. The existence of a synchronized state in a large lattice may be important for applications of polariton condensation in areas such as analog simulation.

The synchronized state we find is not expected for Kuramoto oscillators on a finite-dimensional lattice [5-7]. It arises from the nonodd coupling between the phases, which gives a relevant nonlinear term in the continuum limit. That same form will appear for any system of coupled oscillators $\dot{\theta_{k}}=\omega_{k}+K \sum_{<l>} f\left(\theta_{l}-\theta_{k}\right)$, in which the coupling function $f(x)$ is neither purely even nor odd. That will generally be the case, so that our work implies that other coupled oscillator systems can support synchronized states, notwithstanding disorder in their frequencies. 


\section{ACKNOWLEDGMENTS}

We acknowledge funding from the Irish Research Council under Award No. GOIPG/2019/2824. Some calculations were performed on the Lonsdale Cluster maintained by the Trinity Centre for High Performance Computing. This cluster was funded by grants from Science Foundation Ireland.

\section{APPENDIX A: DERIVATION OF LATTICE MODEL}

We obtain the lattice model, Eq. (2), from the drivendissipative Gross-Pitaevskii equation, Eq. (1), by expressing the wave function as a superposition of wave functions corresponding to the ground state of each well:

$$
\Psi(\mathbf{r}, t)=\sum_{k}^{N} \psi_{k}(t) \phi_{k}(\mathbf{r}),
$$

where the $\phi_{k}(\mathbf{r})$ are normalized. We substitute Eq. (A1) into the Gross-Pitaevskii equation, multiply on the left by $\phi_{l}^{*}(\mathbf{r})$, and integrate over the sample. The wave functions $\phi_{k}$ are localized on each site. Thus, terms involving the direct overlap of wave functions on different sites are exponentially small when the separation of wells is large compared with the size of the $\phi_{k}$. We make the standard simplification of neglecting them [43]. This leads to Eq. (2). The energies in the lattice model $\epsilon_{k}$ are those for the single well. The full tunneling term, corresponding to the linear parts of Eq. (1), is $J_{k l}=-\int \phi_{k}^{*}(\mathbf{r})\left[-(1 / 2 m) \nabla^{2}+V_{0}(\mathbf{r})+i g_{0}(\mathbf{r})\right] \phi_{l}(\mathbf{r}) d \mathbf{r}$. In our model, we neglect the last imaginary part, consistent with dropping other small terms which involve the direct overlap of wave functions on different sites, so that $J_{k l}$ is real. We also retain the tunneling $J_{k l}$ only for nearest-neighbor sites since it decays rapidly with distance. The linear gain on each site is $g_{k}=\int\left|\phi_{k}(\mathbf{r})\right|^{2} g_{0}(\mathbf{r}) d \mathbf{r}$, and the interaction is $U_{k}=$ $\int\left|\phi_{k}(\mathbf{r})\right|^{4} U_{0} d \mathbf{r}$, with a corresponding expression for $\Gamma_{k}$.

As discussed in the main text, the potential we consider consists of a large periodic component, which is the same for each site, with the addition of a smaller random component, which varies from site to site. Since the former is much larger than the latter, the wave functions $\phi_{k}(\mathbf{r})$ will vary little from site to site, and we treat them as identical. Given also that the pump profile is the same for each site- $g_{0}(\mathbf{r})$ has the periodicity of the lattice - then the parameters of the lattice model $g_{k}, U_{k}, \Gamma_{k}$, and $J_{k l}$ are the same for every site.

\section{APPENDIX B: DERIVATION OF PHASE-ONLY MODEL}

We obtain a phase-only model by expressing the wave function on each site in terms of its density and phase.
Substituting this into the lattice version of the driven Gross-Pitaesvkii equation, Eq. (2), and separating the real and imaginary parts gives

$$
\begin{aligned}
& \frac{\dot{n}_{k}}{2 n_{k}}=g-\Gamma n_{k}+J \sum_{<l>} \sqrt{\frac{n_{l}}{n_{k}}} \sin \left(\theta_{l}-\theta_{k}\right), \\
& \dot{\theta}_{k}=\epsilon_{k}+U n_{k}-J \sum_{<l>} \sqrt{\frac{n_{l}}{n_{k}}} \cos \left(\theta_{l}-\theta_{k}\right) .
\end{aligned}
$$

The first two terms of Eq. (B1) imply that, in the absence of tunneling, the occupation of each site relaxes to the steadystate value $g / \Gamma$ with rate $2 g$. We consider the dynamics far enough above threshold for this to be the fastest timescale, allowing us to adiabatically eliminate the occupations by setting $\dot{n}_{k}=0$. We also suppose the condensates are pumped sufficiently far above threshold for the variation in occupation between sites to be relatively small $\delta n_{k l}=n_{l}-n_{k} \ll n_{k}$, so that the square root factors can be replaced with one. Using these conditions to solve Eq. (B1) and substituting into Eq. (B2) gives Eq. (3). A sufficient condition for the siteto-site changes in occupations to be small can be obtained by considering the first two terms on the right-hand side of Eq. (B2), which balance when $\delta n \sim(\sigma / U)$. This is an overestimate of the true $\delta n$ because the final term, which is the kinetic energy, spreads out the wave function. Thus, $\delta n / n \ll 1$ certainly holds when $(\sigma / U) /(g / \Gamma)=\sigma \alpha / g \ll 1$.

\section{APPENDIX C: ESTIMATES OF EXPERIMENTAL PARAMETERS}

For the experiment reported in Ref. [14], the site-to-site variation of the energy, at the scale of a single well, is given as $\sigma=0.03 \mathrm{meV}$. We have estimated the tunneling $J$ by considering a pair of harmonic oscillator potentials, with minima separated by $a$, each with oscillator length $l=\sqrt{\hbar / m \omega}$. Taking $l \approx 3 \mu \mathrm{m}$ and $a \approx 8 \mu \mathrm{m}$, where the synchronization transition was observed, we find $J \approx 7 \mu \mathrm{eV}$, implying $J / \sigma \approx$ 0.2 . However, as the value of $J$ depends exponentially on the form of the potential, there is considerable uncertainty in this estimate.

We could not reliably estimate $\alpha=\Gamma / U=\Gamma_{0} / U_{0}$ from the data reported in Ref. [14], but it can be estimated from other experiments on condensation. Reference [35], using the data from Ref. [8], gives an estimate of 0.3 . We obtain a similar value by relating the model in Ref. [44] and its parameters $\gamma, n_{s}=25000$ and $\kappa=4 \times 10^{-5} \gamma$ to the present Gross-Pitaevskii equation. Comparing the rate equations in the two approaches gives $\Gamma \approx \gamma /\left(2 n_{s}\right)$, while the present $U \approx 2 \kappa$, so that we obtain $\alpha \sim 0.5$.
[1] A. Pikovsky, M. Rosenblum, and J. Kurths, Synchronization: A Universal Concept in Nonlinear Sciences, 1st ed., Cambridge Nonlinear Science Series No. 12 (Cambridge University Press, Cambridge, 2003).

[2] S. H. Strogatz, From Kuramoto to Crawford: Exploring the onset of synchronization in populations of coupled oscillators, Physica D 143, 1 (2000).
[3] Y. Kuramoto, Self-entrainment of a population of coupled nonlinear oscillators, in International Symposium on Mathematical Problems in Theoretical Physics, Lecture Notes in Physics, edited by H. Araki (Springer, Berlin, Heidelberg, 1975) pp. 420-422.

[4] J. A. Acebrón, L. L. Bonilla, C. J. Pérez Vicente, F. Ritort, and R. Spigler, The Kuramoto model: A simple paradigm 
for synchronization phenomena, Rev. Mod. Phys. 77, 137 (2005).

[5] H. Sakaguchi, S. Shinomoto, and Y. Kuramoto, Local and global self-entrainments in oscillator lattices, Prog. Theor. Phys. 77, 1005 (1987).

[6] S. H. Strogatz and R. E. Mirollo, Phase-locking and critical phenomena in lattices of coupled nonlinear oscillators with random intrinsic frequencies, Physica D 31, 143 (1988).

[7] H. Hong, H. Park, and M. Y. Choi, Collective synchronization in spatially extended systems of coupled oscillators with random frequencies, Phys. Rev. E 72, 036217 (2005).

[8] J. Kasprzak, M. Richard, S. Kundermann, A. Baas, P. Jeambrun, J. M. J. Keeling, F. M. Marchetti, M. H. Szymańska, R. André, J. L. Staehli, V. Savona, P. B. Littlewood, B. Deveaud, and L. S. Dang, Bose-Einstein condensation of exciton polaritons, Nature (London) 443, 409 (2006).

[9] I. Carusotto and C. Ciuti, Quantum fluids of light, Rev. Mod. Phys. 85, 299 (2013).

[10] K. G. Lagoudakis, B. Pietka, M. Wouters, R. André, and B. Deveaud-Plédran, Coherent Oscillations in an ExcitonPolariton Josephson Junction, Phys. Rev. Lett. 105, 120403 (2010).

[11] N. G. Berloff, M. Silva, K. Kalinin, A. Askitopoulos, J. D. Töpfer, P. Cilibrizzi, W. Langbein, and P. G. Lagoudakis, Realizing the classical XY Hamiltonian in polariton simulators, Nat. Mater. 16, 1120 (2017).

[12] A. Amo and J. Bloch, Exciton-polaritons in lattices: A nonlinear photonic simulator, C. R. Phys. 17, 934 (2016).

[13] A. Baas, K. G. Lagoudakis, M. Richard, R. André, L. S. Dang, and B. Deveaud-Plédran, Synchronized and Desynchronized Phases of Exciton-Polariton Condensates in the Presence of Disorder, Phys. Rev. Lett. 100, 170401 (2008).

[14] H. Ohadi, Y. del Valle-Inclan Redondo, A. J. Ramsay, Z. Hatzopoulos, T. C. H. Liew, P. R. Eastham, P. G. Savvidis, and J. J. Baumberg, Synchronization crossover of polariton condensates in weakly disordered lattices, Phys. Rev. B 97, 195109 (2018).

[15] M. Wouters, Synchronized and desynchronized phases of coupled nonequilibrium exciton-polariton condensates, Phys. Rev. B 77, 121302(R) (2008).

[16] M. Thunert, A. Janot, H. Franke, C. Sturm, T. Michalsky, M. D. Martín, L. Viña, B. Rosenow, M. Grundmann, and R. Schmidt-Grund, Cavity polariton condensate in a disordered environment, Phys. Rev. B 93, 064203 (2016).

[17] J. D. Töpfer, I. Chatzopoulos, H. Sigurdsson, T. Cookson, Y. G. Rubo, and P. G. Lagoudakis, Engineering spatial coherence in lattices of polariton condensates, Optica 8, 106 (2021).

[18] I. L. Aleiner, B. L. Altshuler, and Y. G. Rubo, Radiative coupling and weak lasing of exciton-polariton condensates, Phys. Rev. B 85, 121301(R) (2012).

[19] H. Ohadi, R. L. Gregory, T. Freegarde, Y. G. Rubo, A. V. Kavokin, N. G. Berloff, and P. G. Lagoudakis, Nontrivial Phase Coupling in Polariton Multiplets, Phys. Rev. X 6, 031032 (2016).

[20] M. P. A. Fisher, P. B. Weichman, G. Grinstein, and D. S. Fisher, Boson localization and the superfluid-insulator transition, Phys. Rev. B 40, 546 (1989).

[21] A. Janot, T. Hyart, P. R. Eastham, and B. Rosenow, Superfluid Stiffness of a Driven Dissipative Condensate with Disorder, Phys. Rev. Lett. 111, 230403 (2013).
[22] L. He, L. M. Sieberer, and S. Diehl, Space-Time Vortex Driven Crossover and Vortex Turbulence Phase Transition in OneDimensional Driven Open Condensates, Phys. Rev. Lett. 118, 085301 (2017).

[23] R. Lauter, A. Mitra, and F. Marquardt, From Kardar-ParisiZhang scaling to explosive desynchronization in arrays of limit-cycle oscillators, Phys. Rev. E 96, 012220 (2017).

[24] V. N. Gladilin, K. Ji, and M. Wouters, Spatial coherence of weakly interacting one-dimensional nonequilibrium bosonic quantum fluids, Phys. Rev. A 90, 023615 (2014).

[25] L. He, L. M. Sieberer, E. Altman, and S. Diehl, Scaling properties of one-dimensional driven-dissipative condensates, Phys. Rev. B 92, 155307 (2015).

[26] E. Altman, L. M. Sieberer, L. Chen, S. Diehl, and J. Toner, Two-Dimensional Superfluidity of Exciton Polaritons Requires Strong Anisotropy, Phys. Rev. X 5, 011017 (2015).

[27] D. Squizzato, L. Canet, and A. Minguzzi, Kardar-Parisi-Zhang universality in the phase distributions of one-dimensional exciton-polaritons, Phys. Rev. B 97, 195453 (2018).

[28] M. Kardar, G. Parisi, and Y.-C. Zhang, Dynamic Scaling of Growing Interfaces, Phys. Rev. Lett. 56, 889 (1986).

[29] W. Ebeling, A. Engel, B. Esser, and R. Feistel, Diffusion and reaction in random media and models of evolution processes, J. Stat. Phys. 37, 369 (1984).

[30] T. Nattermann and W. Renz, Diffusion in a random catalytic environment, polymers in random media, and stochastically growing interfaces, Phys. Rev. A 40, 4675 (1989).

[31] J. Krug and T. Halpin-Healy, Directed polymers in the presence of columnar disorder, J. Phys. I France 3, 2179 (1993).

[32] T. Halpin-Healy and Y.-C. Zhang, Kinetic roughening phenomena, stochastic growth, directed polymers and all that. Aspects of multidisciplinary statistical mechanics, Phys. Rep. 254, 215 (1995).

[33] M. E. Cates and R. C. Ball, Statistics of a polymer in a random potential, with implications for a nonlinear interfacial growth model, J. Phys. France 49, 2009 (1988).

[34] N. Bobrovska and M. Matuszewski, Adiabatic approximation and fluctuations in exciton-polariton condensates, Phys. Rev. B 92, 035311 (2015).

[35] J. Keeling and N. G. Berloff, Spontaneous Rotating Vortex Lattices in a Pumped Decaying Condensate, Phys. Rev. Lett. 100, 250401 (2008).

[36] M. Galbiati, L. Ferrier, D. D. Solnyshkov, D. Tanese, E. Wertz, A. Amo, M. Abbarchi, P. Senellart, I. Sagnes, A. Lemaître, E. Galopin, G. Malpuech, and J. Bloch, Polariton Condensation in Photonic Molecules, Phys. Rev. Lett. 108, 126403 (2012).

[37] I. S. Aranson and L. Kramer, The world of the complex Ginzburg-Landau equation, Rev. Mod. Phys. 74, 99 (2002).

[38] P. Eastham and B. Rosenow, Disorder, synchronization, and phase-locking in nonequilibrium Bose-Einstein condensates, in Universal Themes of Bose-Einstein Condensation (Cambridge University Press, Cambridge, 2017) pp. 462-476.

[39] N. Kopell and G. B. Ermentrout, Symmetry and phaselocking in chains of weakly coupled oscillators, Commun. Pure Appl. Math. 39, 623 (1986).

[40] P. Östborn, Frequency entrainment in long chains of oscillators with random natural frequencies in the weak coupling limit, Phys. Rev. E 70, 016120 (2004). 
[41] L. M. Sieberer, G. Wachtel, E. Altman, and S. Diehl, Lattice duality for the compact Kardar-Parisi-Zhang equation, Phys. Rev. B 94, 104521 (2016).

[42] Y. Imry and S.-K. Ma, Random-Field Instability of the Ordered State of Continuous Symmetry, Phys. Rev. Lett. 35, 1399 (1975).
[43] I. Zapata, F. Sols, and A. J. Leggett, Josephson effect between trapped Bose-Einstein condensates, Phys. Rev. A 57, R28 (1998).

[44] D. M. Whittaker and P. R. Eastham, Coherence properties of the microcavity polariton condensate, Europhys. Lett. 87, 27002 (2009). 